


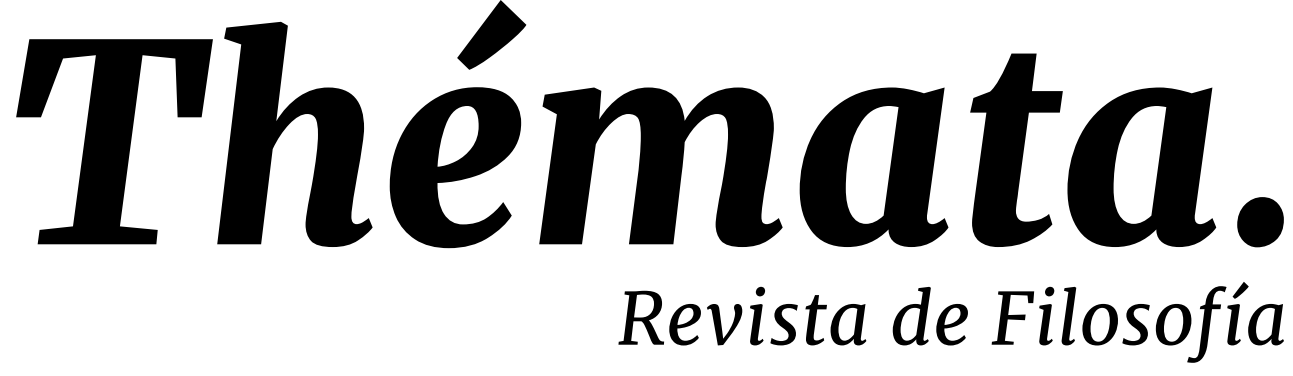

\section{La transmisión filosófica. Pensamiento de Javier Hernández-Pacheco Sanz}

Alejandro Martín Navarro (Coord.)

ESPECIAL THÉMATA

2022

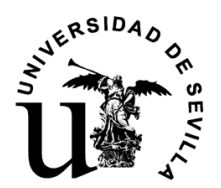

ISSN 0212-8365

e-ISSN 2253-900X

DOI: $10.12795 /$ themata

revistascientificas.us.es/index.php/themata

https://editorial.us.es/es/revistas/themata-revista-de-filosofia 
Thémata. Revista de Filosofía.

Número Extra

Thémata. Revista de Filosofía nace en el año 1983 con la intención de proporcionar a quienes investigan y producen en filosofía un cauce para publicar sus trabajos y fomentar un diálogo abierto sin condicionamientos ideológicos. En sus inicios participaron en el proyecto las Universidades de Murcia, Málaga y Sevilla, pero pronto quedaron como gestores de la revista un grupo de docentes de la Facultad de Filosofía de la Universidad de Sevilla.

Una preocupación constante de sus realizadores ha sido fomentar los planteamientos interdisciplinares. La revista ha estado abierta siempre a colaboradores de todas las latitudes y ha cubierto toda la gama del espectro filosófico, de lo que constituye una buena prueba la extensa nómina de autores que han publicado en sus páginas. En sus páginas pueden encontrarse trabajos de todas las disciplinas filosóficas: Historia de la Filosofía, Metafísica, Gnoseología, Epistemología, Lógica, Ética, Estética, Filosofía Política, Filosofía del Lenguaje, Filosofía de la Mente, Filosofía de la Ciencia, Filosofía de la Historia, Filosofía de la Cultura, etc. También ha querido ser muy flexible a la hora de acoger nuevos proyectos, fomentar discusiones sobre temas controvertidos y abrirse a nuevos valores filosóficos. Por esta razón, los investigadores jóvenes siempre han encontrado bien abiertas las puertas de la revista.

\section{Equipo editorial / Editorial Team Bases de Datos y Repertorios}

Director honorario

Jacinto Choza Armenta

jchoza@us.es

Director

Fernando Infante del Rosal

finfante@us.es

Director Adjunto

José Manuel Sánchez López

themata@us.es

Subdirectores

Jesús Navarro Reyes

jnr@us.es

Inmaculada Murcia Serrano

imurcia@us.es

Jesús de Garay

jgaray@us.es

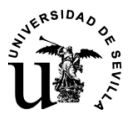

Facultad de Filosofía. Universidad de Sevilla

Departamento de Estética e Historia de la

Filosofía

Departamento de Filosofía y Lógica y Filosofía

de la Ciencia

Departamento de Metafísica y Corrientes

Actuales de la Filosofía, Ética y Filosofía Política

Camilo José Cela s/n, 41018 Sevilla (España)

e-mail: themata@us.es
Bibliográficas internacionales

Emerging Sources Citation Index (Web of

Science Group-Clarivate Analytics)

Dialnet (España)

Francis, Philosophie. INIST-CNRS (France)

Philosopher's Index (Bowling Green, OH, USA)

Directory of Open Access Journals (DOAJ)

Repertoire Bibliographique de Philosophie

(Louvain, Belgique)

Ulrich's Internacional Periodicals Directory

(New York, USA)

DialogJournalNameFinder (Palo Alto, CA, USA)

Periodicals Index Online (Michigan, USA)

Index Copernicus World of Journals

Gale-Cengage Learning-Informe Académico

Academic Journal Database

DULCINEA

Google Scholar

Electra

Bulletin signaletique. Philosophie, CNRS

(France)

Bibliográficas nacionales

ISOC - Filosofía. CINDOC (España)

De evaluación de la calidad de revistas

CARHUS Plus

ERIH PLUS Philosophy (2016)

REDIB

Latindex 2.0

MIAR

CIRC

DICE

Política editorial y directrices para autores/as, al final de la revista.

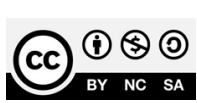


Thémata. Revista de Filosofía.

\section{Consejo Editor / Editorial Board}

\section{ARGENTINA}

Flavia Dezzuto, Universidad Nacional de Córdoba

ALEMANIA

Alberto Ciria, Munich

\section{CANADÁ}

Óscar Moro, University of New Found Land

CHILE

Mariano De la Maza, Universidad Católica de Chile

José Santos Herceg, Universidad de Santiago de Chile

\section{COLOMBIA}

Martha Cecilia Betancur García, Universidad de Caldas

Víctor Hugo Gómez Yepes, Universidad Pontificia Bolivariana

Gustavo Adolfo Muñoz Marín, Universidad Pontificia Bolivariana

\section{ESPAÑA}

Alfonso García Marqués, Universidad de Murcia

Antonio De Diego González, Universidad de Sevilla

Avelina Cecilia Lafuente, Universidad de Sevilla

Carlos Ortiz Landázuri, Universidad de Navarra

Celso Sánchez Capdequí, Universidad Pública de Navarra

Elena Ronzón Fernández, Universidad de Oviedo

Enrique Anrubia, Universidad CEU Cardenal Herrera

Federico Basáñez, Universidad de Sevilla

Fernando Wulff, Universidad de Málaga

Fernando M. Pérez Herranz, Universidad de Alicante

Fernando Pérez-Borbujo, Universitat Pompeu Fabra

Francisco Rodríguez Valls, Universidad de Sevilla

Ildefonso Murillo, Universidad Pontificia de Salamanca

Irene Comins Mingol, Universitat Jaume I Jacinto Rivera de Rosales Chacón, † UNED Joan B. Llinares, Universitat de València Jorge Ayala, Universidad de Zaragoza José Manuel Chillón Lorenzo, Universidad de Valladolid
Juan García González, Universidad de Málaga

Juan José Padial Benticuaga, Universidad de Málaga

Luis Miguel Arroyo Arrayás, Universidad de Huelva

Ma Luz Pintos Peñaranda, Universidad de Santiago de Compostela

Marcelo López Cambronero, Instituto de Filosofía Edith Stein

María del Carmen Paredes, Universidad de Salamanca

Octavi Piulats Riu, Universitat de Barcelona

Óscar Barroso Fernández, Universidad de Granada

Pedro Jesús Teruel, Universitat de València

Ramón Román Alcalá, Universidad de Córdoba

Ricardo Parellada, Universidad Complutense de Madrid

Sonia París Albert, Universitat Jaume I

Tomás Domingo Moratalla, UNED

\section{ESTADOS UNIDOS}

Witold Wolny, University of Virginia)

Thao Theresa Phuong Phan, University of Maryland

\section{REINO UNIDO}

Beatriz Caballero Rodríguez, University of Strathclyde

ITALIA

Luigi Bonanate, Università di Torino

\section{MÉXICO}

Rafael De Gasperín, Instituto Tecnológico de Monterrey

Julio Quesada, Universidad Veracruzana

Adriana Rodríguez Barraza, Universidad Veracruzana

PERÚ

Ananí Gutiérrez Aguilar, Universidad Nacional de San Agustín de Arequipa y Universidad Católica de Santa María

Nicanor Wong Ortiz, Universidad San Ignacio de Loyola

\section{PORTUGAL}

Yolanda Espiña, Universidade Católica Portuguesa

\section{TURQUÍA}

Mehmet Özkan, SETA Foundation for Political, Economic and Social Research 
Thémata. Revista de Filosofía.

\section{Comité Científico Asesor / Advisory Board}

ARGENTINA

Graciela Maturo, Universidad de Buenos Aires - CONICET

Jaime Peire, Universidad Nacional de Tres de Febrero- CONICET

\section{ALEMANIA}

Tomás Gil, Freie Universität Berlin

Fernando Inciarte, $\dagger$ Westfälische WilhelmsUniversität

Otto Saame, † Universität Mainz

\section{BULGARIA}

Lazar Koprinarov, South-West University 'Neofit Rilski'

CHILE

Carla Corduá, Universidad de Chile

Roberto Torreti, Universidad de Chile

COLOMBIA

Carlos Másmela, Universidad de Antioquía Fernando Zalamea, Universidad Nacional de Colombia

\section{ESPAÑA}

Agustín González Gallego, Universitat de Barcelona

Alejandro Llano, Universidad de Navarra Andrés Ortiz-Osés, Universidad de Deusto Ángel D'ors, † Universidad Complutense de Madrid

Antonio Hermosa Andújar, Universidad de Sevilla

Carlos Beorlegui Rodríguez, Universidad de Deusto

Concha Roldán Panadero, Instituto de Filosofía, CCHS-CSIC

Daniel Innerarity Grau, Ikerbasque, Basque Foundation for Science

Francisco Soler, Universidad de Sevilla Ignacio Falgueras, Universidad de Málaga Javier San Martín, UNED

Jesús Arellano Catalán, † Universidad de Sevilla

Joaquín Lomba Fuentes, Universidad de Zaragoza

Jorge Vicente Arregui, † Universidad de Málaga José María Prieto Soler, † Universidad de Sevilla José Rubio, Universidad de Málaga Juan Antonio Estrada Díaz, Universidad de Granada

Juan Arana Cañedo-Argüelles, Universidad de Sevilla
Luis Girón, Universidad Complutense de Madrid

Manuel Fontán Del Junco, Fundación March

Manuel Jiménez Redondo, Universitat de València

Marcelino Rodríguez Donís, Universidad de Sevilla

Miguel García-Baró López, Universidad Pontificia Comillas

Modesto Berciano, Universidad de Oviedo

Pascual Martínez-Freire, Universidad de Málaga

Rafael Alvira, Universidad de Navarra

Teresa Bejarano Fernández, Universidad de Sevilla

Vicente San Félix Vidarte, Universitat de València

\section{ESTADOS UNIDOS}

Lawrence Cahoone, University of Boston

FRANCIA

Nicolás Grimaldi, Université Paris IV-Sorbonne

\section{PARAGUAY}

Mario Ramos Reyes, Universidad Católica de Asunción

\section{REINO UNIDO}

Alexander Broadie, University of Glasgow

ISRAEL

Marcelo Dascal, † Tel Aviv University

ITALIA

Massimo Campanini, Università di Napoli l'Orientale

Maurizio Pagano, Università degli Studi del Piamonte Orientale. Amedeo Avogadro

JAPóN

Juan Masiá, Sophia University, Tokio

\section{MÉXICO}

Jaime Méndez Jiménez, Universidad Veracruzana

Ana Laura Santamaría, Instituto Tecnológico de Monterrey

Héctor Zagal, Universidad Panamericana

VENEZUELA

Seny Hernández Ledezma, Universidad Central de Venezuela

Thémata. Revista de Filosofía agradece la labor realizada desde su inicio por sus anteriores directores: Jacinto Choza, Juan Arana y Jesús de Garay. 


\section{Índice.}

\section{LA TRANSMISIÓN FILOSÓFICA. PENSAMIENTO DE JAVIER HERNÁNDEZ-}

PACHECO SANZ_ESPECIAL THÉMATA

9 El acto filosófico. En recuerdo de Javier Hernández-Pacheco Sanz Fernando Infante del Rosal

14 Presentación

Alejandro Martín Navarro (Coord.)

SEMBLANZAS Y RECUERDOS_JAVIER HERNÁNDEZ-PACHECO SANZ,

TRANSMISIÓN Y ENSEÑANZA DE LA FILOSOFÍA

19 Treinta y siete años con Pacheco. Variaciones sobre el cristianismo Jacinto Choza Armenta

$51 \quad$ Historia de un hombre sabio y bueno Juan Arana Cañedo-Argüelles

$54 \quad$ Una fuerza de la naturaleza José Manuel Sánchez López

58 In Memoriam a Javier Hernández-Pacheco Urbano Ferrer Santos

65 Recordando al profesor Javier Hernández-Pacheco Esperanza María Domínguez Sabido

OBRA Y PENSAMIENTO DE JAVIER HERNÁNDEZ-PACHECO SANZ_ARTÍCULOS

$71 \quad$ Una conversación pendiente Jesús de Garay Suárez-Llanos

87 Un viaje de ida y vuelta: de cómo el refugio se torna apertura. El proyectar(se) como apertura a lo Otro Elisa Fernández Bascones

107 Max Horkheimer y el anhelo de lo completamente Otro. (A propósito de la interpretación de la filosofía de Max Horkheimer de Javier Hernández-Pacheco) José Manuel Panea Márquez 
Thémata. Revista de Filosofía.

124 Javier Hernández-Pacheco: fe y filosofía

Lidia Romero Sánchez

$141 \quad$ La plenitud hacia la que tendemos: historia de una discusión truncada.

Francisco José Soler Gil

161 Tábula gratulatoria.

163 Política editorial.

166 Directrices para autores/as.

ILUSTRACIÓN DE CUBIERTA: Javier Infante 
Thémata. Revista de Filosofía.

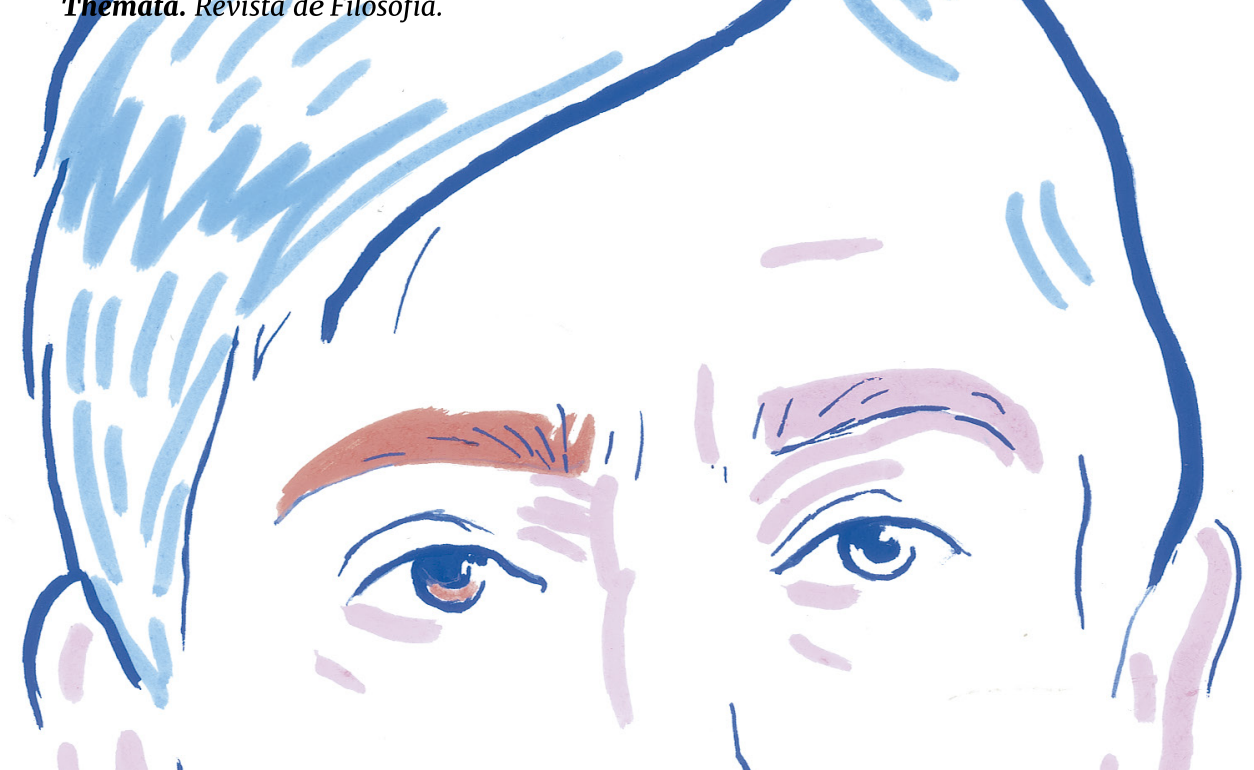




\title{
El acto filosófico. En recuerdo de Javier Hernández-Pacheco Sanz.
}

\author{
Fernando Infante del Rosal' \\ Universidad de Sevilla, España \\ Director de Thémata Revista de Filosofía
}

En la introducción de Hypokeímenon, Javier Hernández-Pacheco afirma que "[... ] la filosofía no es un acto solitario, una reflexión intimista, encerrada en la inmanencia de lo que después se llamará conciencia, sino que desde el primer momento asume la forma de un discurso. Es algo que alguien dice a alguien" (2003 27). En este libro, escrito con zancadas de gigante y atención de tejedora, Hernández-Pacheco pretende rehabilitar lo que en el pensamiento filosófico hay de "dimensionalidad social" (Id.), frente a la dominante del solus ipse, que recorre la historia de la filosofía de Descartes a Husserl, y que identifica el ejercicio de pensar con la autosuficiencia y la intimidad del sujeto, abstraído en sus elaboraciones mentales frente a una estufa o entregado a sus ensoñaciones de paseante solitario.

Hernández-Pacheco señala que el giro lingüístico del pensamiento contemporáneo, primero, y el giro comunicativo, después, enmendarían esta tradición del pensar como acto solitario al evidenciar su "articulación discursiva" (2003 29), su dimensión lingüística e intersubjetiva. Aunque Hernández-Pacheco no lo afirma, tales giros implicarían, por una parte, una comprensión del pensar como ejercido en un terreno que no es, únicamente al menos, el de la conciencia, el de la vida mental; que sale al aire, que se 
incorpora, que se sirve del gesto, de los movimientos del cuerpo. Y, por otra, que el pensamiento se teje como transmisión, que el pensar filosófico se conforma en la cesión del relevo y en la toma del testigo, en la herencia y en un intercambio por entrega (traditio) en el que los que se han ido siguen pensando en el pensamiento de los presentes.

Este cuerpo y esta transmisión recuperados para el pensar filosófico son dos modos de su comunicabilidad - el pensar y todas sus representaciones empiezan a darse como tales cuando son comunicables-. El cuerpo es el país de la palabra viva, pero también el del lenguaje interior y el de las representaciones mentales, que siempre se conjugan con los ojos abiertos o cerrados, así como con dedos volatineros o posados, con pies asentados o inquietos. Leo "Lo que somos es lo que decimos: somos, por así decir, una forma de hablar" (2003 29) y entiendo que Hernández-Pacheco recupera para los fueros del pensamiento el decir y sus modos, pero también veo a Javier, con su característico amago de salto, que le hace levantar primero un pie y luego otro bandeando su enorme presencia como una torre en el momento de enrocarse con un rey, al tiempo que arquea su sonrisa y atrasa su voz hacia un registro más ronco.

En esa presencia movediza, en la encarnación de ese asombro feliz, principio de su filosofía durante mucho tiempo, me comunica su pensar, me aporta significados que no alcanzo con la palabra escrita por él y leída por mí. Aprecio campos que debieron pertenecer al juego de Schiller y al Witz del otro Friedrich, Schlegel. Descubro otro modo de verdad, o la verdad en otro modo. En sus movimientos y en sus gestos mientras habla, en su característica proxemia, en sus trayectos por los intrépidos pasillos de la Facultad de Filosofía o por los más estrechos de una casa no menos laberíntica, en su mohín de extrañeza con el que arruga la frente y hace descender significativamente el inicio de su cabellera, en los objetos de los que se sirve - el libro adosado al corazón; las gafas, destituidas de una mirada en algo nostálgica-, en sus ademanes y sus muecas accedo a otro nivel del sentido.

El cuerpo no solo expresa, tiene también la capacidad de articular, de mover y de dar forma al discurso; y, por eso, tiene también la capacidad de comunicar. Generalmente, en quien se entrega al pensamiento filosófico, tales acciones del cuerpo convergen en los mismos gestos, que sirven para 
modular el pensamiento y para darle parte de su forma comunicativa. Cuando ahora leo a Hernández-Pacheco, a pesar de la distancia, impuesta más por su talento de escritor que por su propia muerte, imagino la presencia de Javier, y los amagos de su figura vienen a enriquecer las representaciones de la mente y los hilos que las atan.

En su obra escrita el cuerpo aparece con frecuencia, generalmente entablillado por Aristóteles y Tomás de Aquino, ligado a temas como la identidad o a la belleza; pero si desarrolló una teoría del cuerpo lo hizo de manera implícita, atravesando obras como la citada. No obstante, como siempre hacía, reparó los dualismos con una dialéctica propia, corporeizada -encabezonada-, que elaboró más allá de Hegel y de Adorno, que ya iban sobrados de obstinación. Así, sobre el cuerpo llegó a afirmar: "La corporalidad se muestra así como el límite de la reflexión, pero por lo mismo que es su medio. Porque sólo por el cuerpo se desarrolla esa actividad refleja que en general llamamos 'vida', y 'alma' o 'espíritu' si la consideramos en absoluto como principio activo de la citada reflexión." (2009 148-149)

El cuerpo, con sus acciones y sus digestiones, está presente en la articulación del pensamiento mismo, es un agente más en la cocina de las ideas. Y es también un modo de transmisión entre quienes han pensado en un mismo espacio y en un mismo tiempo. Es el hermano de la palabra oral y directa, su puesta en escena. La filosofía se transmite tradicionalmente, bien por esa palabra viva, bien por la palabra escrita -el conocido mito de Theuth y Thamus que narra Sócrates en el Fedro expresa esa doble salida-. Las historias de la filosofía alternan momentos en los que la transmisión se ha dado en la presencia del cuerpo y de su voz, con momentos en los que la transferencia se produce más abiertamente a través de los textos. En el primer caso se aplica una empatía entre coetáneos, en el segundo, más bien sinfronismo. Evidentemente, las notas del cuerpo, las inflexiones de la voz y la co-presencia ofrecen una hermenéutica de la motivación que hay que rastrear más arduamente en la palabra escrita, por mucho que ésta garantice otro tipo de precisiones.

Lo primero que aprendí de Javier Hernández-Pacheco fue esa performatividad del pensar filosófico que le da forma interior y forma exterior, que lo mueve por dentro y lo transpone hacia fuera. Esta performatividad define el acto filosófico característico de la enseñanza, el 
modo más genuino de la socialización del pensar y el de mayor persistencia a lo largo de la historia del pensamiento, no sólo occidental; el que, como dice Sócrates, atañe a ese discurso "que se escribe con fundamento en el alma del que aprende" (Platón 276a). No es el de la enseñanza el único acto filosófico, pero sí aquel en el que la presencia física y sensible es convocada de manera más radical, aquel en el que el cuerpo comparece con la responsabilidad de un testigo de cargo.

En Javier Hernández-Pacheco, el acto filosófico se prolongaba en los silencios, necesarios cuando los pasos topaban con un muro y requerían un cambio de rumbo; en su volcar insistentemente la cabeza hacia adelante, como si fingiera dar cabezazos contra ese muro imaginario; en la reticencia hacia cierto desvío en el camino delatada por su mueca de sonrisa cerrada. Todo ello traslucía una intensa vida filosófica, "esa valiente visibilidad de una vida filosófica" de la que hablaba Nietzsche en Schopenhauer como educador (758). En esa intempestiva afirmaba Nietzsche que para él un filosofo era importante en la medida en que estaba en condiciones de dar ejemplo "mediante la vida visible y no meramente con los libros, es decir, como enseñaban los filósofos de Grecia, mediante el rostro, la actitud, el vestido, la comida y las costumbres más que con la palabra o sólo con la escritura" (Id.).

El retrato de cubierta es obra del artista Javier Infante, director del estudio de diseño al que Inma Acosta dedicó años de su trabajo. Esos años están llenos de recuerdos en casa de Inma y Javier Hernández-Pacheco, recuerdos que se alojaron sobre los de otros años. Es probable que, si Javier se viera, advirtiera a Rocío, Eduardo e Ignacio de aquello mismo de lo que avisaba Diderot a su descendencia al escribir sobre el retrato que le hizo Michel Van Loo: "Hijos míos, ya os prevengo, ese personaje no era yo" (Diderot 77). Pero el artista ha visto, a través de sus fotos (su recuerdo personal es lejano), esa dulzura que las maneras de hombre del campo extremeño no hacían más que acrecentar. Tiene el dibujo el aire femenino y levemente jovial de los retratos de los ilustrados, la misma sonrisa etrusca que identificaba su actitud confiada e iconoclasta, aquella con que Maurice Quentin de La Tour se pintó a sí mismo y pintó a los jóvenes Voltaire, D'Alembert y Rousseau; la misma que Ramsay puso en la boca de Hume y Mengs en la de un risueño Carlos III. 
A lo largo de sus casi cuarenta años Thémata ha dedicado algunos números especiales y monográficos a quienes se fueron. Lo ha hecho, no sólo por la justicia que restituye el reconocimiento, o por la obligación moral de la memoria, sino, sobre todo, por aportar algunas notas de la vida filosófica que alumbrase la obra que dejan con las luces de su anterior presencia. En este Especial de Thémata, diferentes amigos y discípulos contribuyen a traer a presencia la vida filosófica de Javier Hernández-Pacheco con significativas semblanzas y estudios que ponen en escena momentos que, más que anécdotas, son verdaderos actos filosóficos, entregados aquí para acompañar a su palabra escrita.

Entre esos amigos están los anteriores directores de esta revista, Jacinto Choza, Juan Arana y Jesús de Garay, que cultivaron durante mucho tiempo un campo en el que muchos tuvimos la fortuna de crecer. Recordamos también a Pilar López de Santamaría y a José María Prieto, que abonaron esos mismos surcos con enorme sabiduría, y a Jacinto Rivera de Rosales, querido miembro de nuestro Consejo Editor.

Bibliografía

Diderot, Denis. Salón de 1767. Trad. Lydia Vázquez. Madrid: Machado Libros. Col. La Balsa de Medusa, 2003.

Hernández-Pacheco, Javier. "De espejos y sistemas nerviosos. la mecánica de la reflexión". Neurofilosofía. Perspectivas Contemporáneas, eds. Concepción Diosdado, Francisco Rodríguez Valls, y Juan Arana. Sevilla/Madrid: Thémata/Plaza y Valdés, 2009. 141-150.

Hernández-Pacheco, Javier. Hypokeímenon. Origen y desarrollo de la tradición filosófica. Madrid: Ediciones Encuentro, 2003.

Nietzsche, Friedrich. "Schopenhauer como educador". Obras completas Vol. I. Escritos de juventud. Edición dirigida por Diego Sánchez Meca. Madrid: Tecnos, 2011.

Platón. Fedro. Trad. Emilio Lledó. Madrid: Gredos, 2010. 


\section{Thémata. \\ Revista de Filosofía}

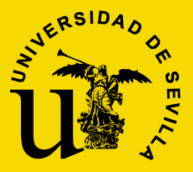

